\title{
Editorial: Multi-scale manipulation toward robotic manufacturing technologies
}

\author{
Hyungpil Moon ${ }^{1}$ - Michael Gauthier ${ }^{2}$
}

(C) Springer-Verlag Berlin Heidelberg 2015

Manipulation is an old traditional subject in robotics. Since the first programmable manipulator was invented more than a half century ago, the manipulation task was the very application of robots. In spite of recent advances in many fields in robotics, manipulation is still intriguing. The main challenges in manipulation come from the complexity of the manipulator and its end effector behavior, uncertain interaction between the manipulator and the environment or the manipulated object, environmental complexities and variability, to potential operational failures. It is more so in $\mathrm{mini} / \mathrm{micro} / \mathrm{nano}$ manipulation due to the lack of dexterity of manipulators and poor measurements in such a scale. The level of automation in micro-manipulation is so far rather limited, not much beyond tele-manipulation and basic visual servoing. Micro-manipulation can be benefitted from developments in macro-scale manipulation. At the same time, handling problems in micro/nano scale particles would inspire researchers in macro-manipulation for further innovation in their methodologies.

The goal of the two special issues about "multi-scale manipulation toward robotic manufacturing technologies" is to present and assess recent advances in manipulation methods in macro-scale which are propitious down to micro-scale robotic manipulation and the efforts in micro-manipulation at the same time.

Hyungpil Moon

hyungpil@me.skku.ac.kr

Michael Gauthier

Michael.gauthier@femto-st.fr

1 School of Mechanical Engineering, Sungkyunkwan University, Suwon, Korea

2 Institut FEMTO-ST, Univ. Bourgogne Franche-Comté/CNRS, Besançon, France
This special issue contains three peer-reviewed papers covering the exciting activities in the field of macro-scale manipulation or manipulator. In Laurent and Moon, a comprehensive review is presented in the subject of the noncontact pneumatic manipulation of flat objects. It provides several open challenges in non-contact pneumatic manipulation. The indirect solid-to-solid contact manipulation such as pneumatic manipulation is expected to be beneficial in micro-scale. Wang, Wu, Kang, and Li reported a smooth trajectory planning for micro-parallel manipulator based on the kinematic transmission property. A high rigidity of the parallel manipulator allows high precision and high speed of movements, which will be beneficial to micro-manipulation. Finally, Kim et al. investigate the object shape reconstruction from object grasping and manipulation. In the later issue, tele-operation and in-hand manipulation issues in microscale will be discussed.

Through these special issue contributions, readers of Journal of Service Robotics will have the chance of grasping the ideas of manipulation in macro-scale that can be bridged toward micro-manipulation, and vice versa.

The Guest Editors would like to acknowledge the journal Editor-in-Chief, the Administrative and Editorial Staff, all the reviewers and, above all, all the authors for their valuable contributions.

Guest editors

Hyungpil Moon (hyungpil@me.skku.ac.kr), Associate Professor, Sungkyunkwan University, Korea

Michael Gauthier (Michael.gauthier@femto-st.fr), Head of the AS2M department, FEMTO-ST Institute, France 